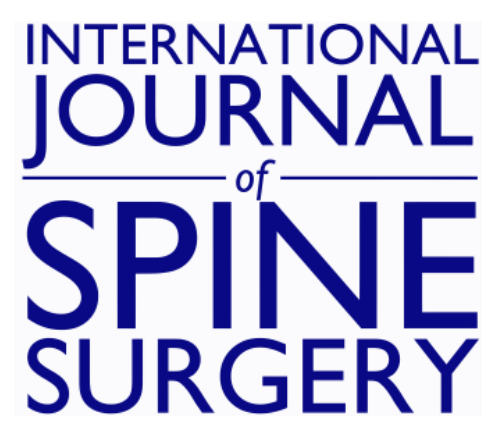

\title{
Early Clinical \& Radiographic Results of NFix II Posterior Dynamic Stabilization System
}

Frank L. Acosta, Jr., Finn Bjarke Christensen, Jeffrey D. Coe, Tae-Ahn Jahng, Scott H. Kitchel, Hans Jörg Meisel, Mark Schnöring, Charles H. Wingo and Christopher P. Ames

Int J Spine Surg 2008, 2 (2) 69-75

doi: https://doi.org/10.1016/SASJ-2007-0121-NT

http://ijssurgery.com/content/2/2/69

This information is current as of April 26, 2023.

Email Alerts Receive free email-alerts when new articles cite this article. Sign up at: http://ijssurgery.com/alerts 


\title{
Early Clinical \& Radiographic Results of NFix II Posterior Dynamic Stabilization System
}

\author{
Frank L. Acosta, Jr., MD, ${ }^{a}$ Finn Bjarke Christensen, $M D$, PhD ${ }^{b}$ Jeffrey D. Coe, $M D,{ }^{c}$ \\ Tae-Ahn Jahng, $M D,{ }^{d}$ Scott H. Kitchel, $M D,{ }^{e}$ Hans Jörg Meisel, $M D, P h D,{ }^{f}$ \\ Mark Schnöring, $M D{ }^{f}$ Charles $H$. Wingo, $M D{ }^{g}$ and Christopher P. Ames, $M D^{a}$
}

\begin{abstract}
Background

Complications associated with rigid posterior instrumented fusions of the lumbar spine include pseudarthrosis, accelerated adjacent-segment disease, hardware failure, and iatrogenic fixed sagittal imbalance. Posterior pedicle screw/rod-based dynamic stabilization systems, in which semirigid rods or cords are used to restrict or control, rather than completely eliminate spinal segmental motion, aim to reduce or eliminate these fusion-associated drawbacks. In this study, we analyzed the early radiographic and clinical outcomes of patients treated with the NFix II System (N Spine, Inc., San Diego, California), a novel pedicle screw/ rod-based system used as a nonfusion posterior dynamic stabilization system, and compared our results to those of similar systems currently in use.
\end{abstract}

\section{Methods}

Seven sites participated in a retrospective assessment of 40 consecutive patients who underwent dynamic stabilization of the lumbar spine with the NFix II System at a single level. (One patient underwent 2 single-level dynamic constructs at noncontiguous levels (L3-4 and L5-S1).) Patients were included based on the presence of spinal stenosis, degenerative spondylolisthesis, adjacent segment degeneration, recurrent disc herniation, symptomatic degenerative disc disease, and degenerative scoliosis requiring dynamic stabilization at 1 level with or without instrumented rigid fusion at a contiguous level. Participants were evaluated preoperatively, with planned postoperative assessments at 3 and 6 weeks ( 1 center assessed patients at 4 weeks), 3 months, 6 months, and 12 months. The primary clinical outcome measures at each assessment were visual analogue scale (VAS) scores to measure back pain, and Oswestry Disability Index (ODI $)^{1}$ scores to measure function. Radiographic outcome measurements included evidence of instrumentation failure and range of motion (ROM) based on postoperative flexion-extension radiographs at 3,6 , and 12 months.

\section{Results}

Forty patients (15males, 25 females) with a mean age of 55 years (range $21-81$ ) were included. Average follow-up was 8.1 months (range 6-12). The mean VAS score improved from 7.6 preoperatively to 3.3 postoperatively $(P<.001)$, and the ODI score from 47.3 to $22.8(P<.001)$. Eighty percent of patients were severely disabled or worse $(\mathrm{ODI} \geq 41)$ preoperatively, which was reduced to $13 \%$ postoperatively. Of the 10 patients with more than 6 months' follow-up, only 4 demonstrated adequate flexion/extension effort. ROM measurements in those 4 patients showed that on average $53 \%$ of preoperative segmental motion was retained at the dynamically stabilized level 6 months postoperatively. There were no instrumentation-related complications.

\section{Conclusions}

Results of this limited study indicate that the NFix II System when used as a nonfusion device for dynamic stabilization produces significant improvements in pain and function at short-term follow-up with outcomes comparable to other dynamic stabilization systems. The use of this system was not associated with an increased risk of instrumentation failure. The small number of patients with postoperative severe disability or worse compares favorably to long-term published data on posterolateral fusion. Lastly, in this small sample, ROM was preserved at 6-month follow-up.

\section{Clinical Relevance}

Posterior pedicle screw/rod dynamic stabilization using the NFix II System seems very effective in improving pain and function scores, at least in the short term (mean postoperative ODI of 22.8). Preservation of ROM is also possible. Longerterm follow-up is necessary to assess sustained clinical improvement, hardware complications, and maintenance in segmental ROM. The NFix II System may be considered an effective alternative to existing dynamic stabilization systems. This device is cleared by the US Food and Drug Administration for use as an adjunct to fusion and has the European CE Marking for use in both fusion and nonfusion applications.

Key Words: Lumbar spine, posterior dynamic stabilization, spinal fusion. SAS Journal. Spring 2008;69-75. DOI: SASJ-2007-0121-NT

${ }^{a}$ Department of Neurological Surgery, University of California, San Francisco; b University Hospital of Aarhus, Aarhus, Denmark; ${ }^{c}$ Silicon Valley Spine Institute, Los Gatos, California; dDepartment of Neurosurgery, Seoul National University, Seoul, Korea; ${ }^{e}$ Orthopedic Spine Associates, Eugene, Oregon; fDepartment of Neurosurgery,BG-Clinic, Bergmannstrost Halle, Germany; gTallahassee Orthopedic Clinic, Tallahassee, Florida 
Address correspondence to Christopher P. Ames, MD, Department of Neurological Surgery, University of California-San Francisco, 505 Parnassus Avenue, M779, Box 0112, San Francisco, CA 94143 (email: amesc@neurosurg.ucsf.edu)

Charles Wingo, MD, owns stock (options, warrants) in and is a consultant to N Spine, Inc. Tah-Ahn Jahng, MD, owns stock in N Spine, Inc. Hans Meisel, MD, is a consultant to N Spine, Inc. and Synthes Spine. Jeffrey Coe, MD, is a consultant to N Spine, Inc.

Institutional Review Board approval was obtained from each institution with study subjects.

\section{INTRODUCTION}

Pedicle screw instrumentation in the lumbar spine has become increasingly popular because, with its use, rigid segmental fixation is often achieved quickly, allowing patients to be mobilized soon after surgery. Modern fusion techniques, including the use of pedicle screws and interbody grafts, have resulted in significant improvements in radiographic evidence of segmental fusion. However, these improvements have not translated into comparable improvements in successful clinical outcomes after lumbar fusion surgery., ${ }^{2,3}$ In addition, the accelerated degeneration of spinal motion segments adjacent to rigidly fused segment(s) has become increasingly recognized as a drawback of spinal fusion procedures. ${ }^{4-7}$ Fixed sagittal imbalance, flat-back syndrome, and pseudarthrosis are also potential complications associated with rigid fusion of the lumbar spine. ${ }^{8}$

It is thought that abnormal load transmission across a degenerated spinal motion segment leads to abnormal segmental motion, altered biomechanics, and subsequently, pain. ${ }^{9,10}$ The goal of dynamic stabilization is to control, rather than eliminate, segmental spinal motion in order to reduce abnormal load transmission across an intervertebral disc and/or facet joint, while avoiding the drawbacks associated with rigid fusion. ${ }^{7}$ This control is accomplished by limiting segmental motion to a range close to normal and/or by preventing the spine from adopting positions or postures that lead to abnormal load transmission. ${ }^{7,11}$ Limiting segmental motion may also be useful to control motion in the iatrogenically destabilized spine; to increase anterior load sharing which augments interbody fusion; and to provide less rigid stabilization in the aging spine. ${ }^{8}$ Additional goals of dynamic stabilization are to cause minimal disruption and to preserve future surgical options.

One of the most extensively used posterior dynamic stabilization systems is the Dynesys Dynamic Stabilization System (Zimmer Spine, Minneapolis, Minnesota), in which pedicle screws are connected across spinal motion segments with nonelastic bands to provide controlled motion. ${ }^{7,12}$ Early clinical outcomes have shown this dynamic stabilization system to be effective in lessening pain and disability at 1-year follow-up in patients with degenerative spondylolisthesis and central or lateral canal stenosis. ${ }^{7}$ The NFix II system is a novel device used in this series as a nonfusion posterior dynamic stabilization system. It incorporates the insertional techniques of traditional pedicle screw/rod-based systems, while providing the potential benefits of dynamic stabilization.

In this study, we describe the early clinical and radiographic experience with the NFix II System. Changes in pain and function were recorded in 40 consecutive patients treated with the system at an average follow-up of 8.1 months. Hardware-related complications were evaluated in all patients. Postoperative range of motion (ROM) was measured in a subset of patients with the longest follow-up $(n=4)$, and results were compared to preoperative values. We hypothesize that the system being evaluated provides comparable clinical improvement in pain and function scores compared to more traditional dynamic stabilization systems, with greater preservation of ROM and without increased hardware-related complications.

\section{MATERIALS AND METHODS \\ Device Information}

The NFix II dynamic stabilization system consists of polyaxial titanium alloy pedicle screws that are fixed to a semirigid polycarbonate urethane-sleeved rod. The composite titanium and polymer sleeve is captured on a solid titanium core by a titanium end cap, which is fixed in place (Figure 1). The rods in this system are low-profile rods that can be used in single- or multi-level applications, and require a relatively short distance between screws of only $9 \mathrm{~mm}$.

\section{Figure 1.}
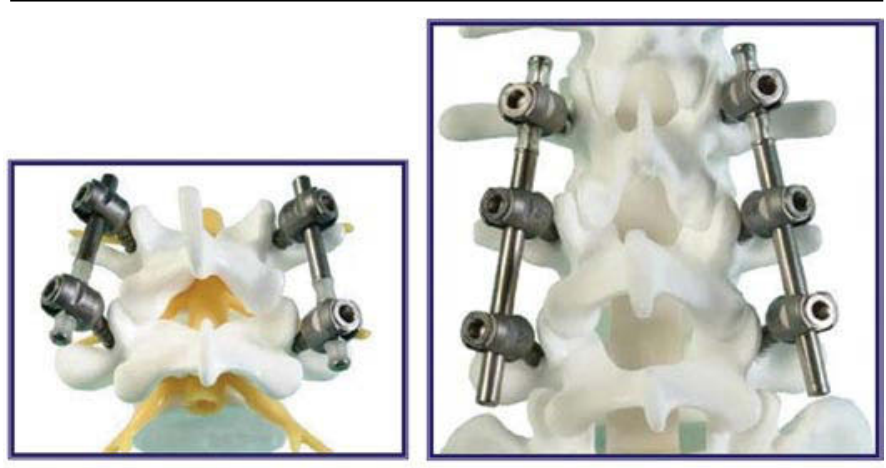

Single-level dynamic construct and 2-level "hybrid" construct.

Prior to FDA clearance of this device as an adjunct to fusion, biomechanical testing was conducted for 10 million cycles according to ASTM standards to assess compression bending, torsion, shear strength, and wear debris. In addition, human cadaveric range of motion studies have demonstrated that approximately $46 \%$ of intact flexion and extension motion and $80 \%$ of lateral bending motion were retained with the NFix II device. ${ }^{13}$ This range of motion is achieved through the controlled pistoning of an integrated dynamic component consisting of a polycarbonate urethane (PCU) spacer and titanium ring, to which a pedicle screw is locked (Figure 2). 
Figure 2.

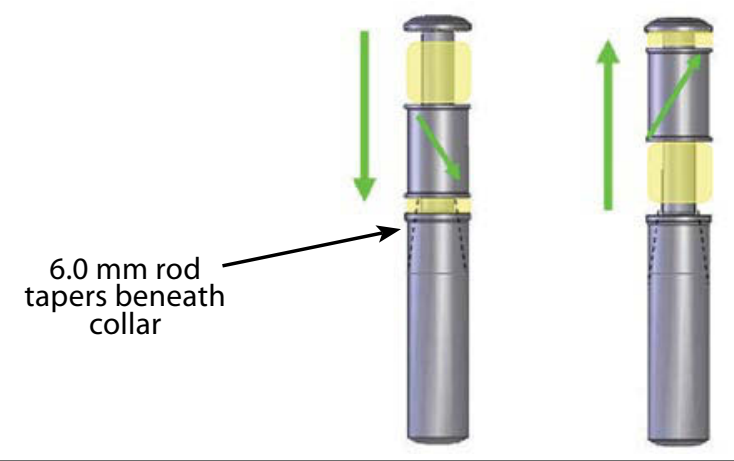

During extension (left) the interpedicular screw distance must decrease, requiring compression of the polycarbonate urethane (PCU) section between the screws. During flexion (right), the interpedicular screw distance must increase, requiring elongation of the device through compression of the opposite PCU section.

\section{Study Design}

This was a non-comparative, retrospective assessment. We present the clinical and radiographic outcomes in 40 consecutive patients who underwent dynamic stabilization of the lumbar spine using the NFix II system as a nonfusion device with a single dynamic segment.

\section{Patient Population}

Seven sites participated in a retrospective assessment of 40 patients who had undergone dynamic stabilization of 1 motion segment of the lumbar spine with the NFix II System between September 2006 and May 2007. The study group consisted of 15 men and 25 women with an average age of 55 years (range $21-81$ ). The average weight was 167 pounds (range 108-295). Ten prior lumbar surgeries were recorded in 8 patients, including 5 discectomies and 5 lumbar fusion procedures (Table 1).

Table 1. Patient Demographics.

\begin{tabular}{ll}
\hline Characteristic & Value (\%) \\
\hline No. patients & 40 \\
Sex Male & $15(38)$ \\
$\quad$ Female & $25(62)$ \\
Age, yrs. (range) & $55(21-81)$ \\
Prior lumbar surgeries & 10 \\
$\quad$ Discectomy & 5 \\
$\quad$ Fusion & 5 \\
\hline
\end{tabular}

Dynamic stabilization was performed for degenerative disc disease (DDD) in 16 patients, spondylolisthesis in 11 patients, fusion-related complications (ie, adjacent segment disease, pseudarthrosis, flat-back syndrome) in 6 patients, lumbar stenosis in 5, and degenerative scoliosis in 2 patients (Table 2).

\section{Preoperative Assessment}

All patients underwent a complete preoperative history and physical examination. Preoperative imaging studies included

\begin{tabular}{lrl}
\hline \multicolumn{2}{l}{ Table 2. Indications for Dynamic Stabilization } \\
\hline Indication & No. of Patients (\%) \\
\hline Degenerative disc disease & 16 & $(40 \%)$ \\
Spondylolisthesis & 11 & $(28 \%)$ \\
Fusion-related sequelae & 6 & $(15 \%)$ \\
Stenosis & 5 & $(12 \%)$ \\
Degenerative scoliosis & 2 & $(5 \%)$ \\
& &
\end{tabular}

anteroposterior and lateral static plain radiographs, as well as dynamic flexion/extension X-rays. Lumbar computed tomography and magnetic resonance imaging were also performed in most cases. Confirmation of symptomatic DDD was based on positive results during provocative discography. All patients completed ODI and VAS questionnaires at all preand postoperative evaluations.

\section{Surgical Procedure}

The studied system construct was implanted using standard surgical procedures for posterior lumbar spinal surgery. Standard midline incisions and subperiosteal retraction of the paraspinal muscles exposing the affected segment were used in most cases. In some cases, however, bilateral paraspinous (Wiltse) incisions were used in order to minimize muscle disruption. If indicated, decompression of the spinal canal was performed first. Insertion of the pedicle screws was performed under radiographic control using a C-arm. Care was taken to place the screws lateral to the facet joints, at $30^{\circ}-45^{\circ}$ lateromedial and parallel to the upper endplate of the corresponding vertebrae.

A special bender was used to contour the rod. This instrument allows bending within a few millimeters of the dynamic section of the rod, while protecting this area from being contoured and potentially damaged. A soft brace was administered after surgery until wound healing had occurred.

\section{Clinical and Radiographic Follow-up}

The patients returned for follow-up evaluations at 3 and 6 weeks ( 1 center assessed patients at 4 weeks), 3 months, 6 months, and 12 months. A thorough neurologic examination was performed, as were static and dynamic lumbar plain films to evaluate for ROM and hardware integrity. ODI and VAS scores were also collected.

\section{RESULTS}

Average follow-up was 8.1 months (range 6-12 months).

\section{Perioperative Data}

The levels treated with the evaluated dynamic stabilization system are presented in Figure 3. All patients were treated with a construct that provided a single dynamic level. One patient underwent 2 single-level dynamic constructs at noncontiguous levels (L3-4 and L5-S1). Instrumented rigid fusion was performed at contiguous levels in 27 patients (the socalled "hybrid construct"), placed either cephalad or caudal to 


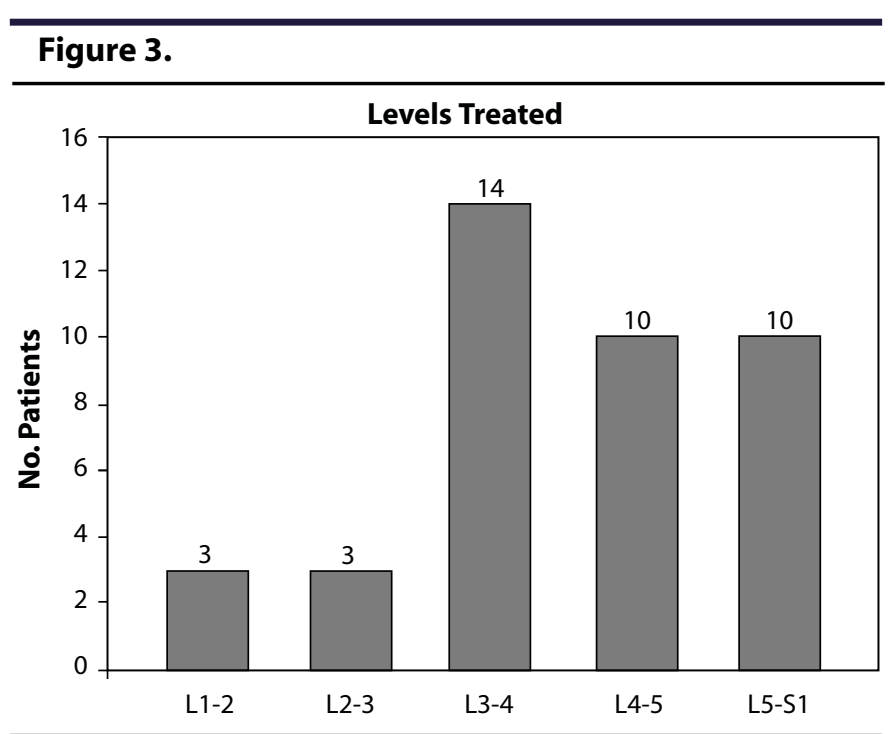

Summary of number of levels treated with dynamic stabilization.

the dynamic level. A single-level rigid fusion was performed in 22 patients; a 2-level rigid fusion in 3 patients; and a 3-level rigid fusion in 2 patients. Dynamic stabilization was used at the contiguous superior level in $21(78 \%)$ patients, and in 6 $(22 \%)$ patients at the inferior end of the fused segment(s).

\section{Clinical Outcomes}

Improvements in pain (VAS) scores for the 40 patients after surgery are presented in Figure 4. The mean VAS score improved from

Figure 4.

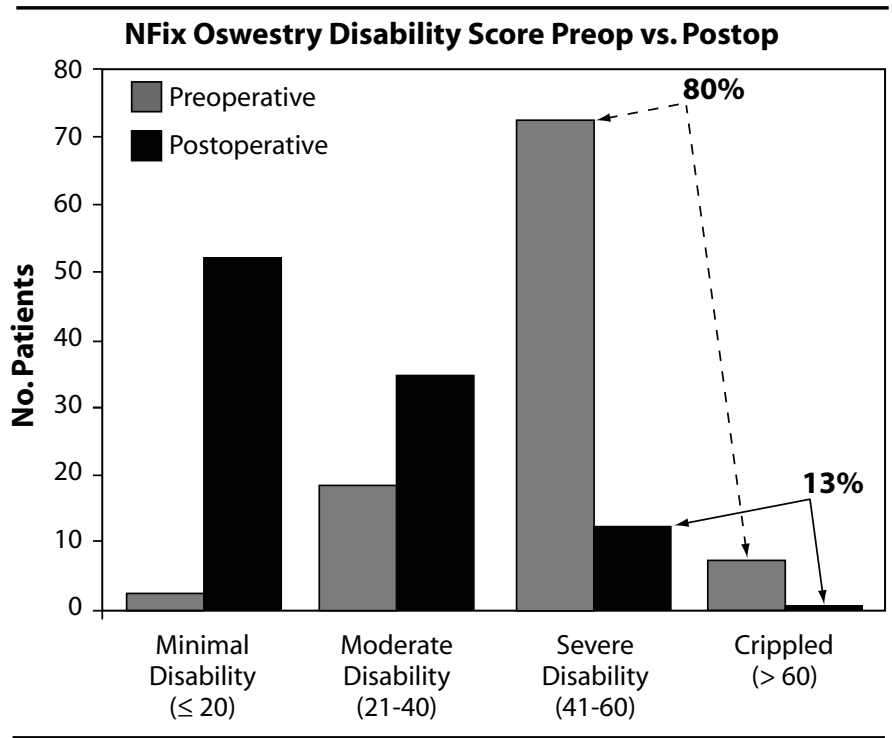

Summary of number of levels treated with dynamic stabilization.

7.6 preoperatively to 3.3 postoperatively $(P<.001)$. Functional status as measured by the ODI score also showed significant improvement (Figure 5). The ODI score is measured from 0-100\%, in which $80-100 \%$ means bedridden/exaggerating

\section{Figure 5.}

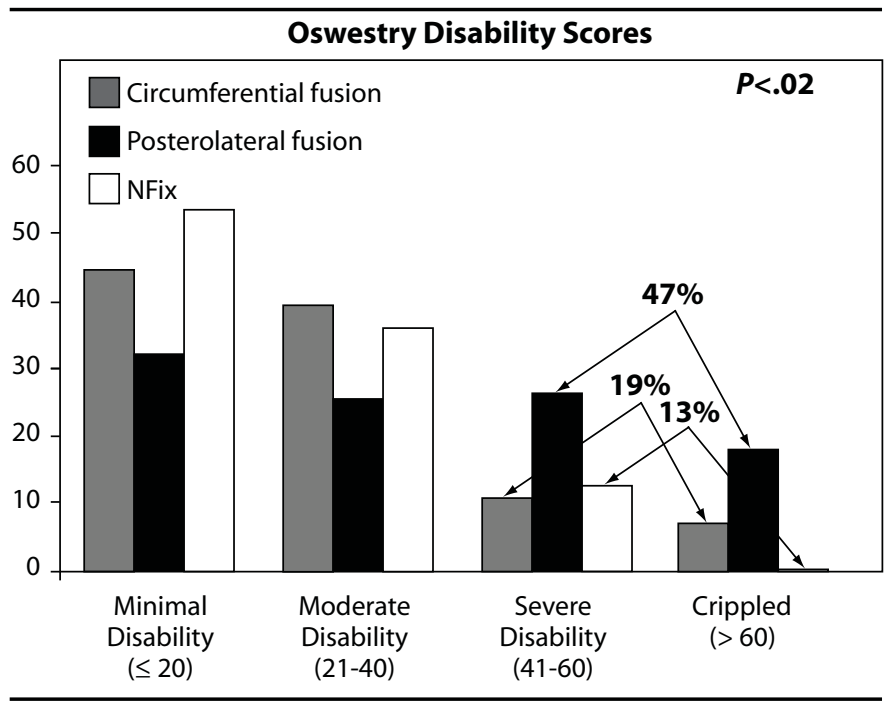

Comparison of improvements in ODI after circumferential fusion, posterolateral fusion, and dynamic stabilization in the current study.

symptoms; $61-80 \%$ means crippled; $41-60 \%$ means severely disabled; $21-40 \%$ means moderate disability; and $0-20 \%$

means minimal disability. The average preoperative ODI of

47.3 improved to 22.8 postoperatively $(P<.001)$. A long-term

\section{Figure 6.}

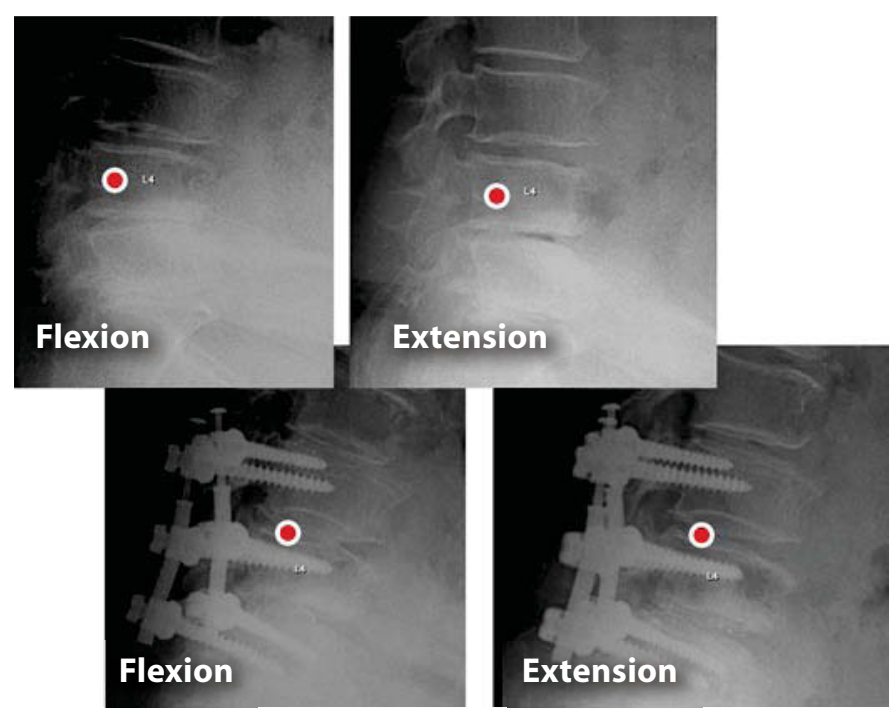

Fifty percent retained ROM. Preoperatively L3-L4:6.8 ROM. Postoperatively L3-4: $3.4^{\circ}$ ROM. Note: red dot indicates center of rotation.

prospective randomized clinical study by Videbaek et al., ${ }^{17}$

comparing posterolateral fusions to circumferential fusions

demonstrated that $47 \%$ of the posterolateral fusion patients had severe disability or worse at follow-up, compared to $19 \%$ of 
the circumferential fusion group. In the postoperative group of patients who received the evaluated system, only $13 \%$ of patients had severe disability or worse. As a preliminary observation, these data compare favorably to the posterolateral fusion group (Figure 6).

\section{Radiographic Measurements}

All patients underwent postoperative static and dynamic plain radiographic imaging which demonstrated no evidence of hardware loosening or rod fracture at 3, 6, and 12 months. ROM measurements were obtained in the 6-month and longer follow-

\section{Figure 7.}

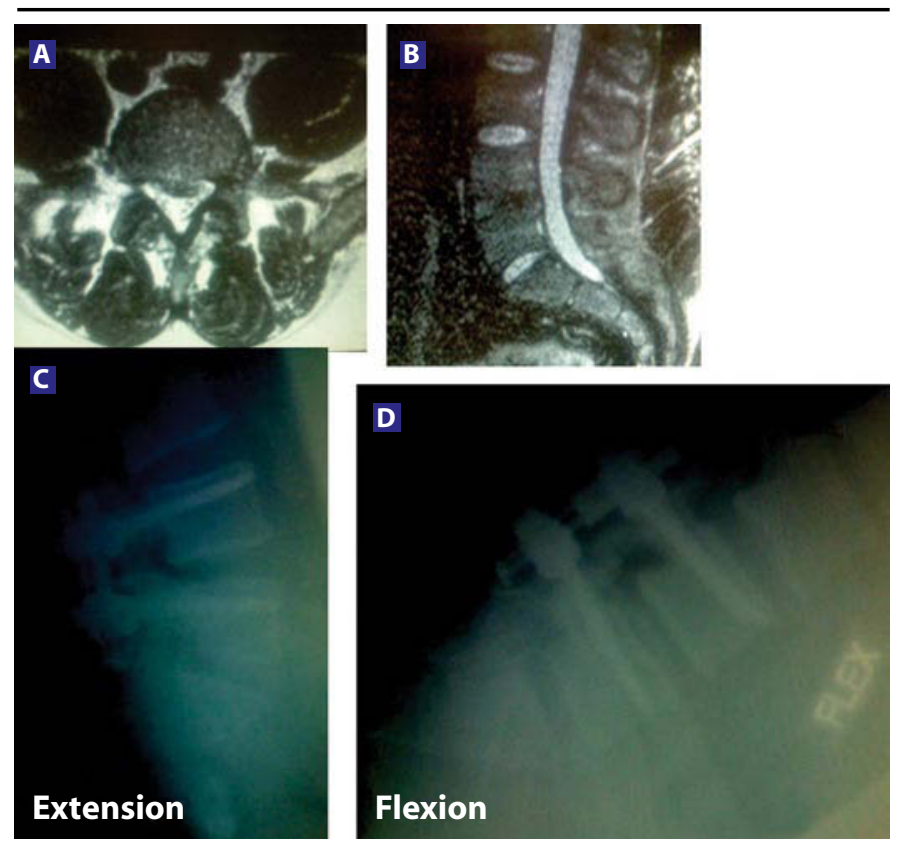

Thirty-nine-year-old male, 309 pounds. One-year history of lower back and left buttocks discomfort. Single functional spine unit (FSU) involved. Unresponsive to conservative care. Treated with hemi-laminectomy at L4-5 and single-level dynamic stabilization. Axial (A) and saggital (B) T2weighted MR images showing degeneration of the L4-5 intervertebral disc. Three-month postoperative films of extension (C) and flexion (D) demonstrate motion at the dynamically stabilized level.

up group. Of the 10 patients with more than 6 months' followup, only 4 patients demonstrated adequate flexion/extension effort as assessed by Medical Metrics, Inc. of Houston, Texas; however, this patient group demonstrated an average retention of $53 \%$ of preoperative ROM $\left(6.4^{\circ}\right.$ preoperatively versus $3.4^{\circ}$ postoperatively) (Figure 7), as determined by Medical Metrics, Inc.

\section{Complications}

There were no significant intra- or perioperative complications related to the device. One patient required additional surgery after the index procedure to replace a loose locking cap on the pedicle screw. One patient required re-operation due to severe osteoporosis, cage migration, and retrolisthesis at the fusion level. The dynamic part of the system showed no problems in this patient. One patient broke both sacral screws in the rigidly fused segment, possibly related to a fall. Reintervention was not required, and the dynamically fixed segment was not involved.

There was no evidence of rod-related complications in any of the patients, and no screw loosening was observed. Finally, no patient experienced new or worsened symptoms at postoperative follow-up, with the exception of the re-operated severely osteoporotic patient noted above.

\section{DISCUSSION}

Modern instrumentation-based techniques for fusion of the lumbar spine, including pedicle screw-rod fixation, interbody grafting, and various osteoinductive and osteoconductive allograft materials, have resulted in significant improvements in radiographic spinal fusion. Nevertheless, improvements in the clinical outcome for patients with lumbar spinal disease after "successful" fusion procedures have not been as robust. ${ }^{2,3}$ The complete elimination of motion, therefore, across a degenerated spinal segment may not be the standard treatment for patients with painful degenerative disorders of the lumbar spine.

The rationale for dynamic stabilization of the lumbar spine lies in the concept of controlling, rather than eliminating, motion across a spinal segment to normal or near-normal ranges. This controlled motion is thought to prevent abnormal loading across a degenerated facet or intervertebral disc, thereby restoring normal biomechanical segmental motion and/or preventing the spine from adopting a position in which abnormal loading may occur. ${ }^{7}$ Moreover, the fact that segmental motion is relatively preserved may prevent the occurrence of various rigid fusion-related sequelae, including adjacent segment disease, pseudarthrosis, and flat-back syndrome.

Indeed, experience with the Dynesys system, the most extensively used dynamic stabilization system to-date, has shown improvements in pain and function scores at least as good as those after lumbar fusion procedures with up to 3year follow-up. ${ }^{7,12,14-18}$ In particular, Welch et al. ${ }^{18}$ presented preliminary clinical outcomes of dynamic stabilization with the Dynesys spinal system as part of a multicenter, randomized, prospective Food and Drug Administration (FDA) investigational device exemption (IDE) clinical trial. The preoperative mean ODI score was $55.6 \%$ compared to the 12 -month follow-up mean score of $26.3 \%$. Thus, there is evidence supporting the concept that dynamic stabilization of the degenerated lumbar spine is effective in the clinical setting.

However, there are several practical drawbacks to existing posterior pedicle screw-based dynamic stabilization systems. For example, the Dynesys system is fairly complex to use intraoperatively and is available for use only with special screws. In the event that subsequent conversion to a rigid fusion is necessary, the screws must be removed and replaced 
with traditional pedicle screws. The Dynesys device cannot be used in a true hybrid configuration, nor does it allow for elongation. The inability to increase the interpedicular distance through device elongation significantly limits the potential for motion in flexion. Consequently, this device is considered to be quite stiff in flexion. Finally, while the Zimmer DTO Implant system allows for hybridization and/or conversion, it is still necessary to remove the Dynesys screws to convert to a rigid rod at the dynamic level.

The NFix II System represents an effort to improve on current dynamic stabilization systems. This system, when used as a nonfusion posterior dynamic stabilization system, provides segmental stabilization through the use of a more traditional titanium rod with an integrated dynamic component, instead of employing cables or cords. This makes the intraoperative placement of the evaluated system similar to more traditional current generation of top-loading pedicle screw/rod-based systems, which have been in use for several years. The integrated dynamic component consists of a polycarbonate urethane (PCU) spacer that allows controlled pistoning during flexion, extension, and lateral bending. Furthermore, the angular motion between this spacer and the central titanium core rod contributes significantly to the angular deflection capacity of the device by absorbing $75 \%$ of bending stresses. The system can also be elongated and is available for use in true hybrid configurations.

Biomechanically, the study system acts like a solid rod in axial rotation, providing resistance against shear, while allowing for the necessary increase and decrease in interpedicular distance (elongation and compression) that is required for flexion, extension, and lateral bending. Finally, the study system may allow for a more physiologic range of motion than the previously available systems. Although ROM was accurately measured in only 4 patients in our study, an average of $53 \%$ of preoperative ROM was retained in this group (postoperative $\mathrm{ROM}=3.4^{\circ}$ ), while only $33 \%$ of total intact flexion-extension ROM was demonstrated in studies of the previous system (postoperative $\mathrm{ROM}=1.9^{\circ}$ ). ${ }^{19}$ This possible increase in ROM may theoretically reduce abnormal loading on adjacent facets and intervertebral discs, as well as reduce or eliminate the possibility of screw loosening.

Device rigidity has been demonstrated to impact the strains at the bone/screw interface such that stiffer devices transmit greater stresses to this interface. A study by Moumene et al. ${ }^{20}$ concluded that, "Using a flexible rod in pedicle screw based PDS [posterior dynamic stabilization] systems helps control the motion of the spine with low risk of screw loosening. Alternatively, the Dynesys System's stiffness is equivalent to that of a solid rod. Screw loosening is expected with Dynesys, as the maximum loosening torque of $2.6 \mathrm{Nm}$ is greater than the reported maximum insertional torque of $1.96 \mathrm{Nm}$."

Lastly, the increased ROM with the study system may allow for increased load-sharing by interbody grafts when used as an adjunct to fusion. It should be noted that conclusions based on the hybrid data we have presented are limited by the fact that we did not have a matched "non-hybrid" cohort by the same surgeon.

\section{CONCLUSIONS}

Posterior dynamic stabilization has been shown to be effective in providing improvements in function and pain for patients with degenerative diseases of the lumbar spine. Nevertheless, previously available posterior dynamic stabilization systems left much to be improved upon. We have found that the NFix II system, when used as a nonfusion dynamic stabilization system, provides improvements in pain and function scores comparable to those found in use of the Dynesys system, although our follow-up is much shorter. In addition, these preliminary data compare favorably to the ODI scores of the posterolateral fusion group in a randomized prospective clinical trial, as the percentage of NFix II patients with severe disability or worse was only $13 \%$ (compared to $47 \%$ in the posterolateral fusion group). Finally, partial preservation of normal ROM (53\%) has been demonstrated in a small number of patients in our cohort; there have been no rodrelated adverse events; and the option of later converting a dynamically stabilized segment to a fusion is preserved.

This manuscript was submitted October 29, 2007, and accepted for publication February 29, 2008.

\section{REFERENCES}

1. Fairbank JC, Pynsent PB. The Oswestry Disability Index. Spine. 2000;25(22):2940-2952; discussion 2952.

2. Boos N, Webb JK. Pedicle screw fixation in spinal disorders: a European view. Eur Spine J. 1997;6(1):2-18.

3. Resnick DK, Choudhri TF, Dailey AT, et al. Guidelines for the performance of fusion procedures for degenerative disease of the lumbar spine. Part 5: correlation between radiographic and functional outcome. J Neurosurg. 2005;2(6):658-661.

4. Chou WY, Hsu CJ, Chang WN, Wong CY. Adjacent segment degeneration after lumbar spinal posterolateral fusion with instrumentation in elderly patients. Arch Orthop Trauma Surg. 2002;122(1):39-43.

5. Etebar S, Cahill DW. Risk factors for adjacent-segment failure following lumbar fixation with rigid instrumentation for degenerative instability. $J$ Neurosurg. 1999;90(2 Suppl):163-169.

6. Markwalder TM, Wenger M. Adjacent-segment morbidity. J Neurosurg. 2002;96(1 Suppl):139-140.

7. Welch WC, Cheng BC, Awad TE, et al. Clinical outcomes of the Dynesys dynamic neutralization system: 1-year preliminary results. Neurosurg Focus. 2007;22(1):E8.

8. Khoueir P, Kim KA, Wang MY. Classification of posterior dynamic stabilization devices. Neurosurg Focus. 2007;22(1):E3.

9. Mandigo CE, Sampath P, Kaiser MG. Posterior dynamic stabilization of the lumbar spine: pedicle based stabilization with the AccuFlex rod system. Neurosurg Focus. 2007;22(1):E9.

10. Yamamoto I, Panjabi MM, Crisco T, Oxland T. Three-dimensional movements of the whole lumbar spine and lumbosacral joint. Spine. 1989;14(11):1256-1260. 
11. Mulholland RC, Sengupta DK. Rationale, principles and experimental evaluation of the concept of soft stabilization. Eur Spine J. 2002;11 Suppl 2:S198-205.

12. Grob D, Benini A, Junge A, Mannion AF. Clinical experience with the Dynesys semirigid fixation system for the lumbar spine: surgical and patient-oriented outcome in 50 cases after an average of 2 years. Spine. 2005;30(3):324-331.

13. Yim J, Crawford NR. Motion characteristics of the NFlexTM pedicle screw based dynamic stabilization system: A human cadaver study. In: American Association of Neurological Surgeons/Congress of Neurological Surgeons Section on Disorders of the Spine and Peripheral Nerves; 2008; Orlando, Florida.

14. Bordes-Monmeneu M, Bordes-Garcia V, Rodrigo-Baeza F, Saez D. [System of dynamic neutralization in the lumbar spine: experience on 94 cases.]. Neurocirugia (Astur). 2005;16(6):499-506.

15. Putzier M, Schneider SV, Funk JF, Tohtz SW, Perka C. The surgical treatment of the lumbar disc prolapse: nucleotomy with additional transpedicular dynamic stabilization versus nucleotomy alone. Spine. 2005;30(5):E109-14.

16. Schnake KJ, Schaeren S, Jeanneret B. Dynamic stabilization in addition to decompression for lumbar spinal stenosis with degenerative spondylolisthesis. Spine. 2006;31(4):442-449.

17. Schwarzenbach O, Berlemann U, Stoll TM, Dubois G. Posterior dynamic stabilization systems: DYNESYS. Orthop Clin North Am. 2005;36(3):363-372.

18. Stoll TM, Dubois G, Schwarzenbach O. The dynamic neutralization system for the spine: a multi-center study of a novel non-fusion system. Eur Spine J. 2002;11 Suppl 2:S170-178.

19. Davis R. Dynamic stabilization: a preliminary in situ quantitative dynamic analysis of flexion extension radiographs from the US IDE trial. In: Proceedings from the Spine Arthroplasty Society; 2007; Berlin, Germany.

20. Moumene M, Mathis W, Harms J, Albert T, Labrom R, Wang M. Assessment of screw loosening with pedicle screw-based posterior dynamic stabilization systems. In: The International Meeting on Advanced Spinal Techniques; 2007; Paradise Island, Bahamas. 